\title{
System Simulation as Part of Systems Engineering for Headlamp and Pedal Systems based on the Modeling Language Modelica
}

\author{
Heinz-Theo Mammen*, Phillip Limbach, Thorsten Maschkio \\ HELLA GmbH \& Co. KGaA, Rixbecker Straße 75, 59552 Lippstadt; *Heinz-Theo.Mammen@hella.com
}

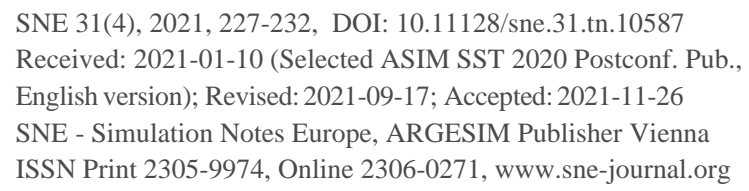

SNE 31(4), 2021, 227-232, DOI: 10.11128/sne.31.tn.10587 Received: 2021-01-10 (Selected ASIM SST 2020 Postconf. Pub., English version); Revised: 2021-09-17; Accepted: 2021-11-26 SNE - Simulation Notes Europe, ARGESIM Publisher Vienna ISSN Print 2305-9974, Online 2306-0271, www.sne-journal.org

Abstract. Based on two application examples, i.e. a DCactuator and a brake pedal system, the analysis of this article demonstrates, which synergies can be achieved by applying system simulation. The first example, focussing on the simulation of the temperature behaviour of a DCactuator to identy critical operating conditions, reveals an effort reduction by a factor of 4 in comparison to measurements. The second example, a brake pedal system, shows an approach of contact modeling between rigid bodies in Modelica, in order to analyse the kinematic movement of the pedal and its force-path characteristics. In this case, the original effort of model creation has been reduced by a factor of $10 \mathrm{~s}$.

Beside the increase of efficiency, the respective de-sign concepts could be optimized concerning the friction and temperature behaviour according to the specifications.

\section{Introduction}

Due to rising complexity of automotive systems and subsystems, system simulation is becoming more and more important to continuously analyse and verify the interdisciplinary system behaviour along the product development process.

Therefore, system models for various subsystems and components have been developed and validated at HELLA in recent years. In this article two examples, i.e. a DC actuator of a mechatronic headlamp module and a brake pedal system, are presented, aiming at the development of applicable models for an efficient and effective system development and optimization.

Considering temperature behaviour, the DC-actuator model includes:
- the control unit,

- the electrical adjustment unit (DC-motor)

- the gear.

The brake pedal system consists of:

- the joint system,

- the position detection unit

- the reset mechanism.

The basic model structure has been automatically derived from the CAD design including its kinematics via direct coupling and extended by modelling the friction behaviour, foot force, return springs and contact surfaces.

For the development of the models, including models for contact surfaces, the modeling language Modelica and the simulator Dymola [1] [2] have been applied.

\section{Model of the DC-Actuator}

The increasing utilization of mechatronic components in the automotive industry can be exemplarily shown on headlamps. While previously simple light sources in combination with reflectors provided a static illumination of the street, nowadays complex mechatronic headlamp systems provide a variety of functions (such as dynamic cornering light, automatic levelling, and many more) depending on the driving conditions. Since such mechatronic systems are getting more and more complex, a holistic development approach is necessary, which can be supported by accompanying modeling. Regarding the example of the DC-actuator, the development and application of a system model aims at increasing system understanding and efficiency at the same time, in particular for the analysis of critical operating conditions, such as blocking of the motor.

The DC-actuator is a subsystem of the mechatronic system headlamp and consists of the control unit, the electrical adjustment unit (motor), the gear as well as the drive shaft. 
FiguFigure 1 exemplarily shows a DC-actuator of a mechatronic headlamp module.

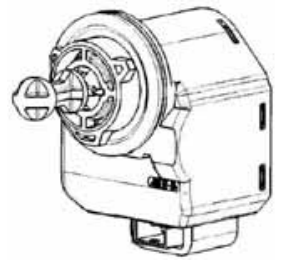

Figure 1: DC-actuator of a mechatronic headlamp modul.

In the following sections, these individual model elements are described.

\subsection{Control Unit of the DC-Actuator}

The schematic diagram of the control unit of the DC-actuator is shown in Figure 2 and essentially consists of two half bridges and a logic module to control them.

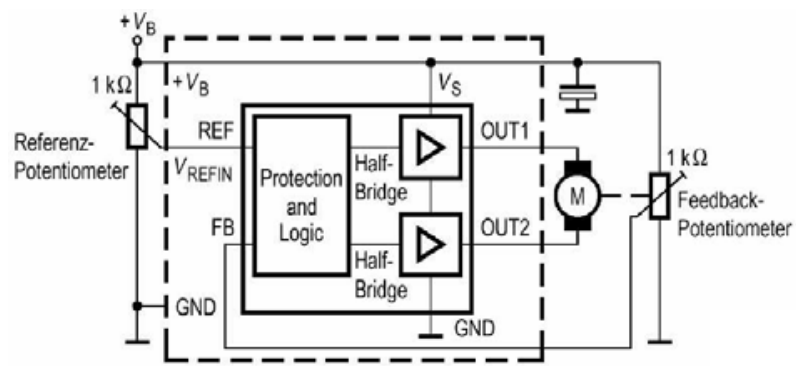

Figure 2: Schematic diagram of the DC-actuator.

The corresponding model of the control unit is shown in

FiguFigure 3. It is modeled in a hierarchical manner, i.e. the half bridges shown in Figure 2 are part of the IC TLE 4209G (see Figure 3).

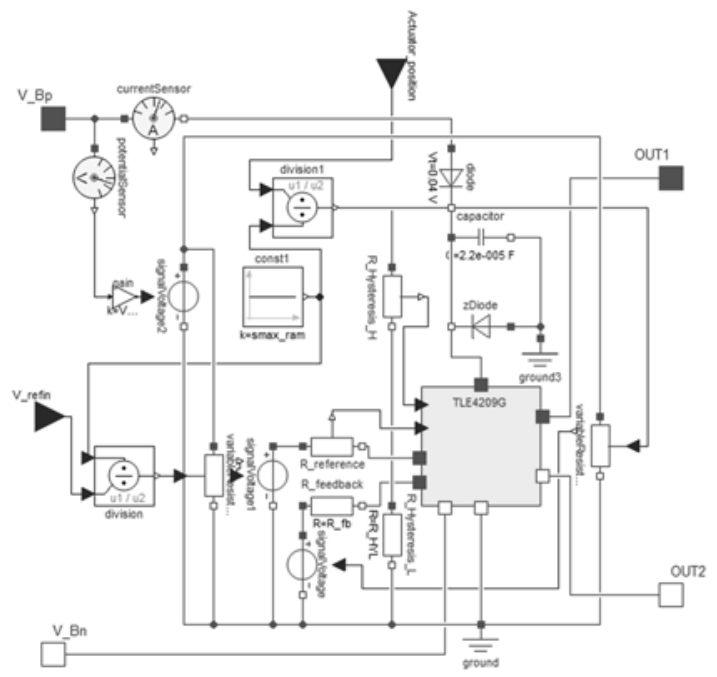

Figure 3: Control unit model of the DC-actuator.

\subsection{Motor Model of the DC-Actuator}

The motor model of the DC-actuator represents the electrical, mechanical and thermal behaviour. Regarding the latter one, on the one hand, the environment temperature and on the other hand the self heating during operation are considered. The Dymola/Modelica motor model is shown in Figure 4.

The accurate prediction of the thermal behaviour requires the description of the commutation characteristics. The motor consists of three windings, which are powered by a commutator. In case of blocking (malfunction), the motor must withstand a continuous current feed over a long period of time. Dependent on the type of blocking, the temperature may quickly increase to over $200^{\circ} \mathrm{C}$ due to self heating, so that the critical motor temperature is exceeded, which in turn leads to failure of the actuator.

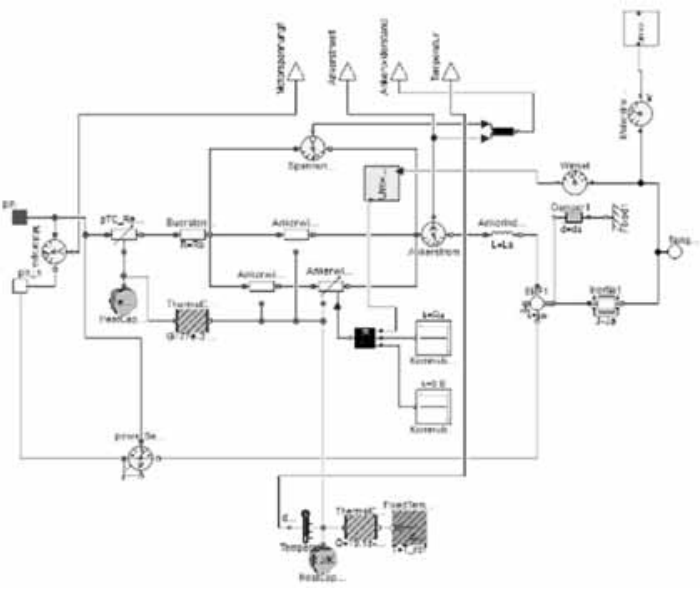

Figure 4: Motor model of the DC-actuator.

The analysis aims at the prediction of the temperature behaviour of different motors in case of a blocking. A distinction is made between two blocking cases, i.e. 1/3 and $2 / 3$ blocking. In case of the $1 / 3$ blocking, both brushes are each on one vane of the commutator. In case of the $2 / 3$ blocking, one of the two brushes is between two vanes, bridging them. This results in two states of armature resistance in case of blocking as shown in Figure 5.

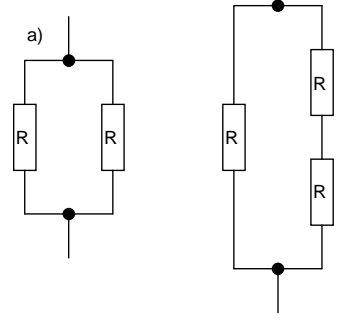

Figure 5: a) 2/3 blocking

b) $1 / 3$ blocking. 
For a $2 / 3$ blocking, the total armature resistance amount to $R_{t o t}=1 / 2 \mathrm{R}$ and for $1 / 3$ blocking to $R_{t o t}=2 / 3 R$. Both cases can be adjusted separately in the model, in order to evaluate the related temperature behaviour in case of blocking explicitely.

\subsection{Gear Model of the DC-Actuator}

The gear model describes the behaviour of a worm gear, as well as the stops limiting both directions of the actuator. In addition, the friction characteristics such as the Coulomb and Stribeck forces are modeled. Summarizing, this model includes parameters for the gear ratio, both stops and friction. The gear model is shown in Figure 6.

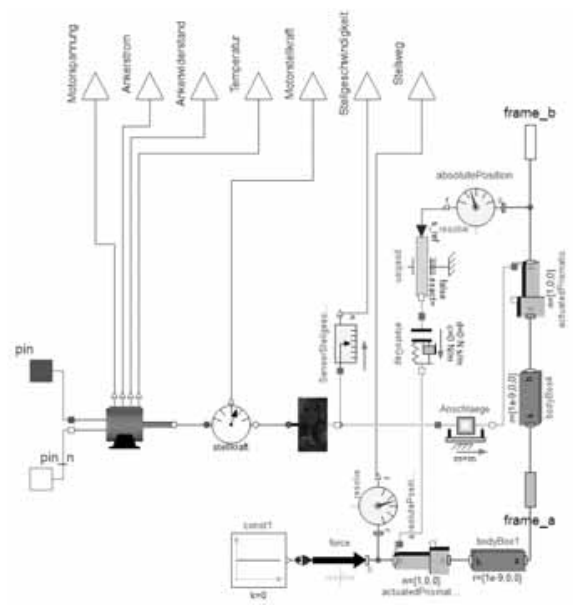

Figure 6: Gear model of the DC-actuator (including DC-motor).

\subsection{Model Validation}

The validation of the model plays a major role to ensure that the model is applicable without restrictions for all investigations along the development process.

Parameterization of the model can be done via specification sheets or measurements. With regard to motors, specification sheets are often not sufficient to define all necessary parameters. Hence, measurements need to be performed, which are usually extensive in terms of costs and time. In this case, relevant motor parameters have been evaluated from specification sheet data, while temperature characteristics have been determined by means of measurements in a climate chamber. These measurements have been performed on three operating conditions, i.e. rotary motion, $1 / 3$ blocking and $2 / 3$ blocking. Following from that, the necessary model parameters have been determined and implemented in the model.
As a next step, the model is validated for several test circuits by means of comparison of simulation results and measurements, respectively. An exemplary test circuit is shown in Figure 7.

Figure 8 exemplarily shows the comparison of the predicted and measured armature current of the DC-motor.

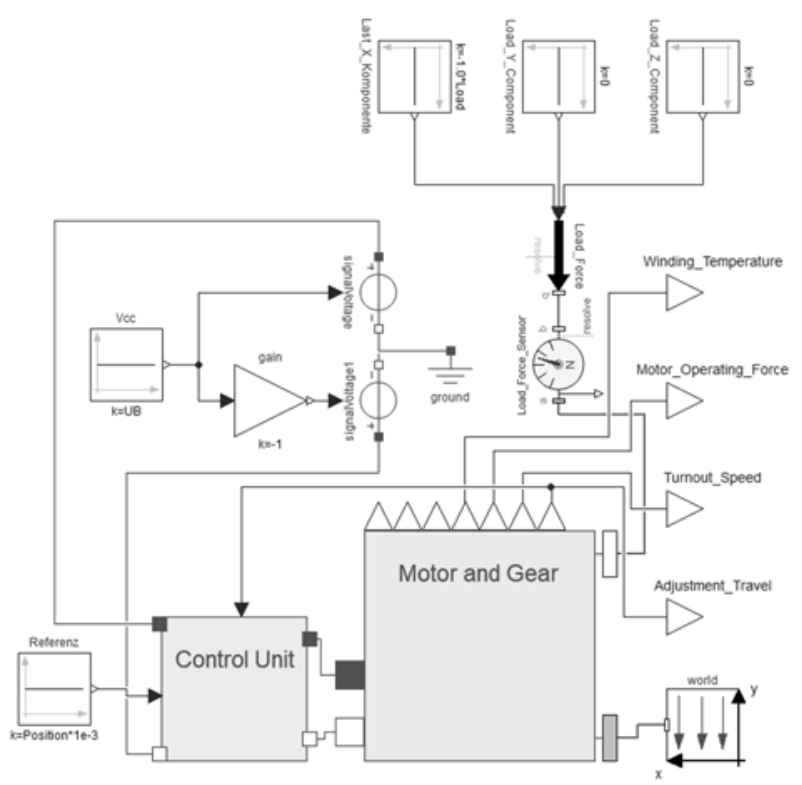

Figure 7: Exemplary test circuit for model validation.

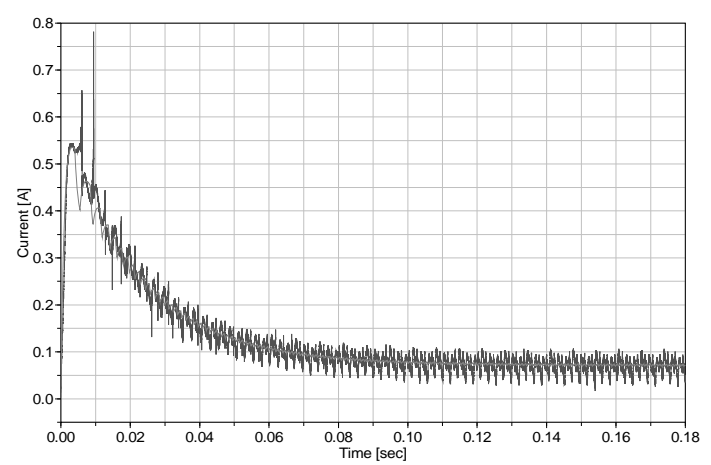

Figure 8: Armature current of the DC-motor model Comparison between measurement (blue) and simulation (red).

The trend of the measured armature current of the DCmotor is satisfactorily reproduced by the simulation, which has been also confirmed for additional setups. Hence, the model is valid to be utilized in the context of product development.

Analysing the effort for prototype hardware development versus simulation reveals a ratio of $4: 1$, i.e. the effort for hardware development is four times higher than for simulation. This leads to the conclusion that by applying simulation methods, the development effort in the respective concept phases can be decreased significantly. 


\section{Model of a Brake Pedal System}

Three different types of pedals can be distinguished in a vehicle, i.e. the brake pedal, the clutch pedal as well as the accelerator pedal.

Since these pedal systems are no longer mechanically connected to the systems to be operated (e.g. connection of the gas pedal to the motor via rope system), todays pedal systems are restricted to various requirements. Hence, beside the pedal arm, these pedal systems consist of friction elements, sensors and springs, in order to imitate closely the requested haptic feedback. Due to the rising complexity of interacting components, a system model has been developed aiming at a virtual prediction of the force-path behaviour, which includes the demand for an efficient contact modeling. An exemplary pedal system is shown in Figure 9.

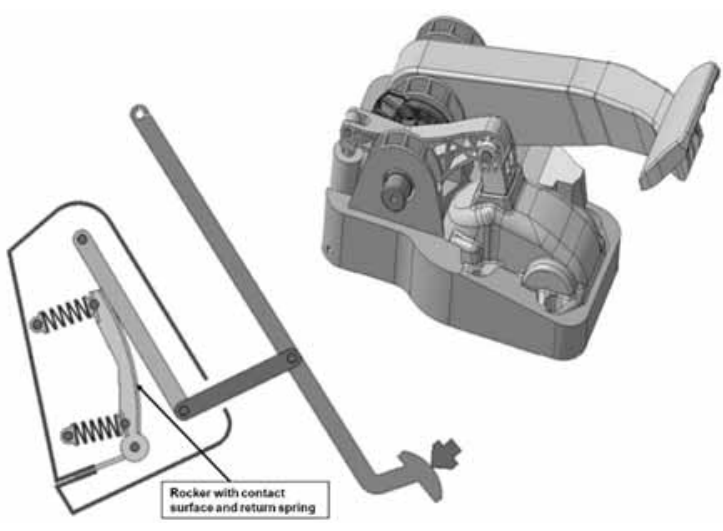

Figure 9: Example of a brake pedal system.

This brake pedal essentially consists of a four-bar linkage, a rocker including return spring, a cam shape defining the contact surface and a friction element. The contact surface forms the interface between the four-bar linkage and the rocker. The geometric shape of the contact surface contributes to the force-path behaviour, so that an appropriate shape design is necessary to satisfy the customer requirements. Most of the above-mentioned pedal elements can be modeled by using Modelica standard elements, while modeling the contact surface is a particular challenge. Hence, this is described in more detail. One possible contact modeling approach in Dymola is available via the Idealized Contact Library [3].

\subsection{Description of a Contact Surface}

In a system simulation model, bodies are usually assumed to be rigid. This assumption is valid if the kinematics are in the focus of investigation.
In this case it is sufficient to describe the body by the location of its center of gravity as well as its mass and inertia moments within the center of gravity. The body expansion is not considered. However, regarding contact phenomena, both, modeling elastic bodies as well as defining the surface are mandatory.

For the description of simple geometries, such as rectangles, cylinders or spheres, the Idealized Contact Library provides one "surface" block, respectively, that describes surface dimensions as well as its orientation in the coordinate system. The latest release additionally provides also blocks for the description of ellipsoids and other convex bodies.

The "surface" block represents a thin surface with no mass, which can be connected to a rigid body via a "frame" interface.

The calculation of the contact force is performed in a "contact" block. Necessary information of the contact surface is transfered via a "contact" interface. Beside defining the body-fixed coordinate system of the surface, geometric information of the contact surface and (in the latest release) the surface type are transferred.

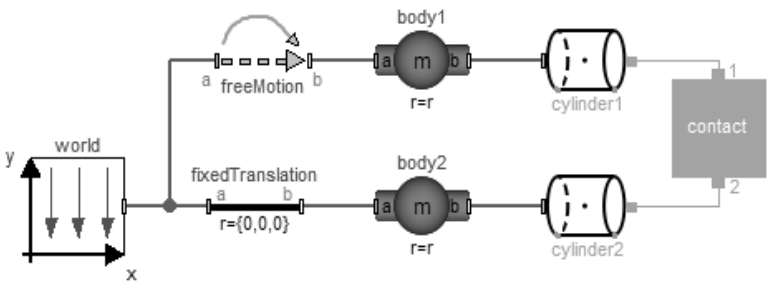

Figure 10: Schematics of a simple contact model with two cylinders.

Figure 10 shows an exemplary model for two cylinders. The blocks "cylinder1" and "cylinder2" describe the surfaces, connected via a "contact" block (orange). More complex geometries can be assembled by a parallel connection of individual contact surfaces. In this case, each contact pair must be connected via a "contact" block [3]

\subsection{Surface Modeling via Analytical Functions}

The approach to describe the cam shape via analytical functions results from the necessity of a variable curvature, which must be tangential and constant in curvature in the region of interest.

Third degree polynomial function. According to this approach, the geometry is described by one or more polynomial functions, which satisfy the necessary geometrical conditions at their intersection points. 
A third degree polynomial function is defined by

$$
\begin{array}{r}
f(z)=a_{3}\left(z-z_{0}\right)^{3}+a_{2}\left(z-z_{0}\right)^{2} \\
+a_{1}\left(z-z_{0}\right)+a_{0}
\end{array}
$$

and is clearly defined by determining the coefficients $a_{i}$ as well as the parameter $z_{0}$. If the coordinates of two points on the function and the first and second derivatives are known, the unknown variables can be determined by

$$
\begin{gathered}
a_{0}=f\left(z_{1}\right)-a_{1}\left(z-z_{0}\right)-a_{2}\left(z-z_{0}\right)^{2} \\
-a_{3}\left(z-z_{0}\right)^{3} \\
a_{1}=\frac{f\left(z_{2}\right)-a_{0}-a_{2}\left(z-z_{0}\right)^{2}-a_{3}\left(z-z_{0}\right)^{3}}{\left(z-z_{0}\right)} \\
a_{2}=\frac{f^{\prime}\left(z_{1}\right)-a_{1}-3 a_{3}\left(z-z_{0}\right)^{2}}{2\left(z-z_{0}\right)} \\
a_{3}=\frac{f^{\prime \prime}\left(z_{1}\right)-2 a_{2}}{6\left(z-z_{0}\right)}
\end{gathered}
$$

The parameter $z_{0}$ is defined as zero. If there is an intersection to another polynomial function, which is tangential and constant in curvature in the intersection point, the results of the first and second derivatives in this point are equal and thereby known, while only the coordinates of two points on the second function need to be specified. The model to describe a polynomial function is integrated in the Modelica model.

The contact surface model and the model components for determining the contact point on the ellipsoidal surface are partially shown inFigure 11 .

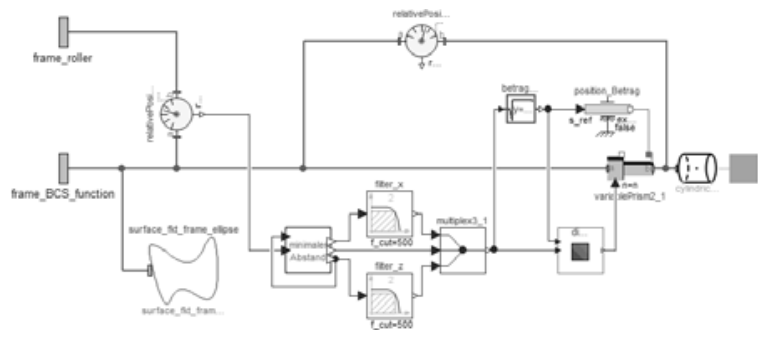

Figure 11: Schematics of the model for calculation of the contact point on the ellipsoidal surface.

To calculate the contact point on the cam shape, the Newton method is implemented.

If the polynomial function is defined within a limited range only, calculation of the minimal distance is performed only in this range by defining thresholds. This is realized by setting the position of the contact point equal to its respective threshold value in case the threshold is exceeded.

One single polynomial function is not sufficient to describe the cam shape and to achieve the demanded pedal behaviour along the entire path.
Hence, the model is assembled by several functions and successively approximated to the demanded behaviour.

Realization of the cam shape. First, the cam shape is described by a single polynomial function and adapted to the demanded behaviour of pedal force at the start of the displacement in accordance with the specified preload and implemented spring parameters of the rocker.

If the force deviates from the reference values at higher pedal angles, the position of the contact point for the respective pedal angle is determined and defined as intersection point for the successive function. Next, the second point of the successive function is specified, in order to achieve the demanded behaviour also for the second section. This is continued successively, until the cam shape is completely described.

The defined surface shape can be directly considered for the design of the geometry in CATIA [4]. This leads to a significant reduction of design steps, prototype development and measurements. In comparison to a decoupled approach, the effort of model creation has been reduced by a factor of 10 .

\subsection{Validation of the System Model}

The overall system model basically consists of 4 main categories, as shown in Figure 12.

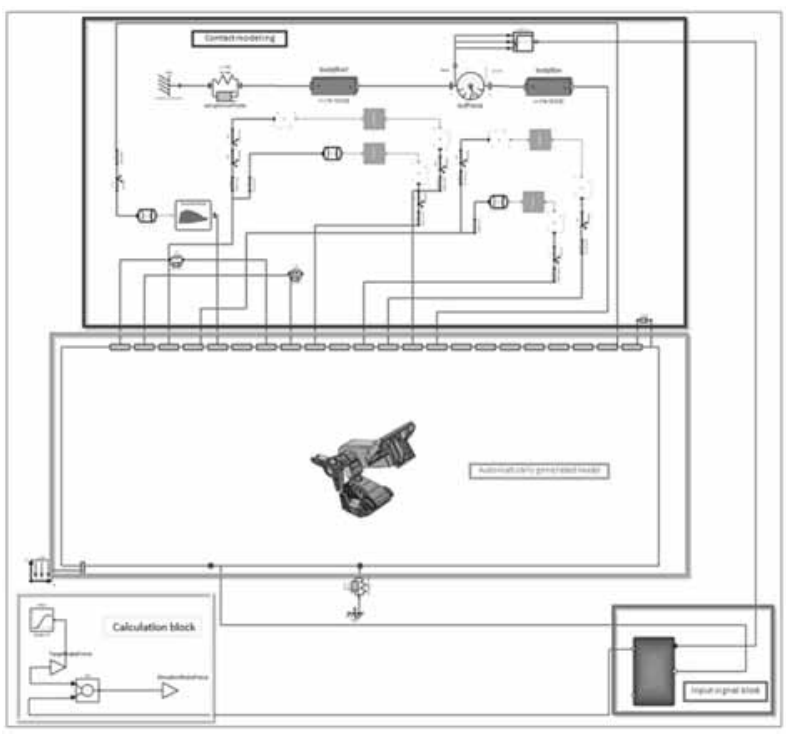

Figure 12: System model of the pedal system.

These are the pedal geometry, which is automatically translated from the CATIA design, a component for modeling the restoring force and the contact surface between the four-bar linkage and the rocker, as well as components for modeling the foot force and friction within the pedal. 
The simulation result of this model is shown in Figure 13 in terms of the pedal force as a function of the pedal angle in comparison to the requested characteristics.

In case of return, the simulation result slightly deviates from the desired behaviour. However, the general trend can be reproduced. By increasing the friction value (which is not verified by measurements), the spread of the hysteresis can be increased.

By adjusting the cam shape, the behaviour can be accurately predicted. For a pedal angle of $32^{\circ}$ the return force is smaller than the demanded minimum. Going towards a smaller pedal angle, the desired behaviour can be satisfactorily achieved.

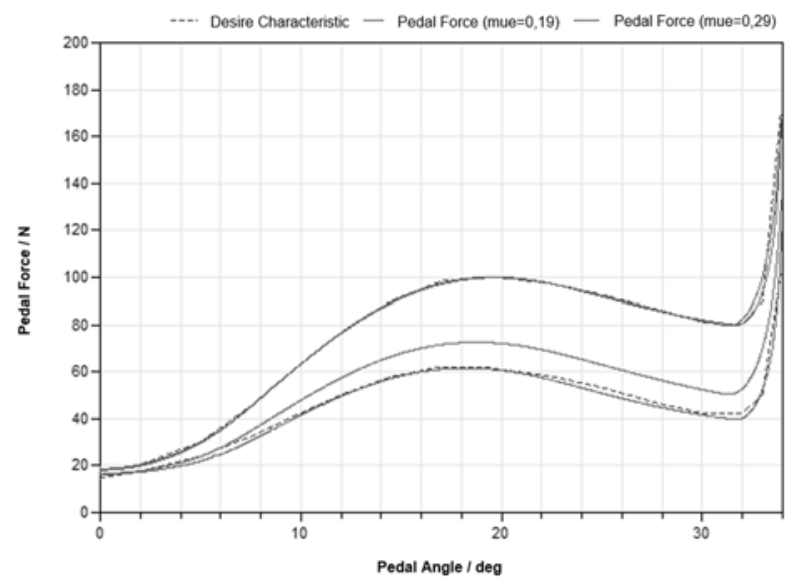

Figure 13: Pedal force as a function of the pedal angle for varying friction values.

\section{Summary}

The application of system simulation delivers an effective possibility to verify the system behaviour along all phases of product development, leading to a reduction of cost and time (e.g. by reduction of prototypes), and moreover, to gain more information about the system, which is not accessible in prototype measurements (e.g. force measurements in encapsulated systems).

The examples presented in this paper clearly demonstrate that the system behaviour including critical operating conditions can be predicted and analyzed via system simulation, leading to a more comprehensive understanding about the system. This is also supported by animations, which simplify interpretation and communication between different stakeholders.

Moreover, such an approach can support on detecting and avoiding failures, which often have tremendous consequences, in early phases of development. In addition, the simulation approach offers the possibility to exactly reproduce scenarios and results, so that the impact of changes can be analyzed and different variants and concepts can be compared more accurately.

\section{Literature}

[1] Fritzson P. Object-Oriented Modeling and Simulation with Modelica 2.1. John Wilex \& Sons, New York 2004.

[2] DyMOLA: Multi-Engineering Modeling and Simulation.

www.dynasim.se, www.dymola.com.

[3] Oestersötebier F, Wang P, Trächtler A. A Modelica Contact Library for Idealized Simulation of Independently Defined Contact Surfaces. Paderborn.

[4] Dassault Systemes: 3DExperience Platform https://www.3ds.com/de/ueber-3ds/3dexperienceplattform/ 